\title{
On nestedness analyses: rethinking matrix temperature and anti-nestedness
}

\author{
Mário Almeida-Neto, Paulo R. Guimarães Jr and Thomas M. Lewinsohn \\ M. Almeida-Neto (marioeco@gmail.com) and T. M. Lewinsohn, Laboratório de Interaçôes Insetos-Plantas, Depto Zoologia, Inst. de \\ Biologia, Univ. Estadual de Campinas, 13083-970, Campinas SP, Brazil. - MAN and P. R. Guimarães Jr., Curso de Pós-graduação \\ em Ecologia, Inst. de Biologia, Univ. Estadual de Campinas. PRG also at: Estación Biológica de Doñana, CSIC, Apdo. 1056, \\ ES-41080 Sevilla, Spain.
}

\begin{abstract}
The analysis of nested structures in sets of species assemblages across different sites or in networks of interspecific interactions has become common practice in ecological studies. Although new analyses and metrics have been proposed, few studies have scrutinized the concepts that subtend nestedness analysis. We note two important conceptual problems that can lead to terminological inconsistencies and flawed interpretations. First, the thermodynamic analogy that underlies the most common metric of nestedness, matrix temperature, is flawed and has led some authors to incorrect interpretations. Second, the term "anti-nestedness" is a potential source of confusion and inconsistencies. We review four concepts for anti-nestedness and examine how distinct they are. "Anti-nested" matrices, i.e. less nested than expected by chance, may result from different ecological processes and show distinct structural patterns. Thus, there is no single unequivocal opposite of nestedness to be represented as "anti-nestedness". A more profitable approach is to designate and test each distinct non-nested pattern according to its specific assumptions and mechanistic hypotheses.
\end{abstract}

In community ecology and biogeography, ecological systems usually are described as presence-absence matrices, often called incidence matrices (Diamond 1975, Gotelli 2000), or as networks (Pimm 1982, Pascual and Dunne 2005). By tradition, incidence matrices are used to investigate distributional patterns of species composition among different habitats or islands (Diamond 1975), whereas a network representation has been adopted in studies of species interactions (Pimm 1982, Jordano 1987). However, incidence matrices and bipartite networks are interchangeable representations for identical structures (Harary 1969, Lewinsohn et al. 2006) and measurements associated with one representation can be directly applied to the other. In this context, the analysis of nestedness, widely used to characterize matrices of species distributions, was recently applied to characterize ecological networks (Bascompte et al. 2003, Dupont et al. 2003, Ollerton et al. 2003, Guimarães et al. 2006, Lewinsohn et al. 2006).
The ecological concept of nestedness stems from Darlington (1957), but only after its development by Patterson and Atmar (1986) and Patterson (1987), was it formally defined and popularized among ecologists. According to Patterson and Atmar (1986), species assemblages are nested if the species present in species-poor sites are a proper subset of those present in species-rich sites. Perfect nestedness occurs when all species-poor sites are proper subsets of the assemblages found in species-rich sites (Fig. 1a-b), whereas a maximally nested matrix is a special case of a perfectly nested matrix with 50\% fill in which presences occupy an upper-left triangle (Fig. 1a). Similarly, bipartite networks are nested if those species with fewer interactions are preferentially associated with a subset of species that interact with the most connected ones (Bascompte et al. 2003). The concept of nestedness has thus been used to indicate both distributional and interactive descriptions of community structure in ecological studies. Here, we use "presence" to designate 
Perfect nestedness

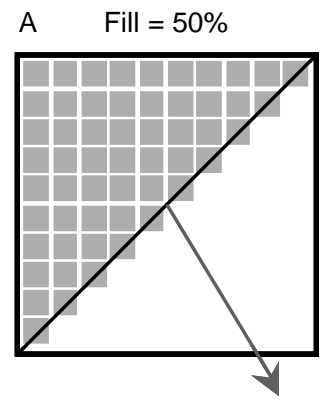

Boundary line
Imperfect nestedness

B Fill $=19 \%$
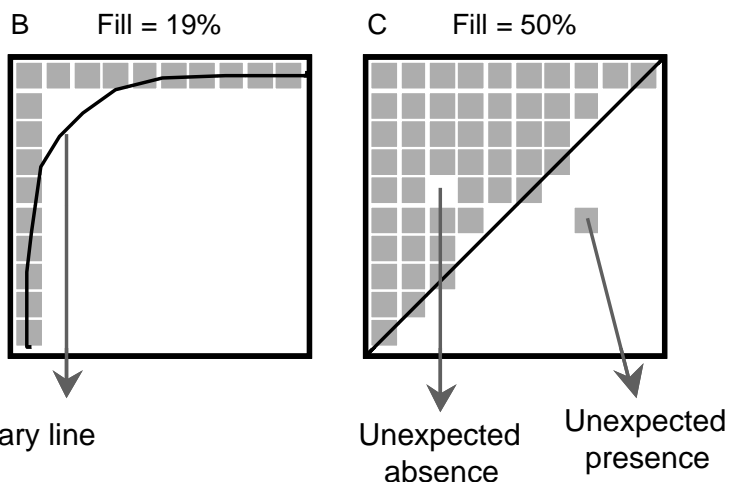

Fig. 1. Three examples of nested matrices. Matrices A and B illustrate the extreme models of perfect nestedness, a maximally informative matrix and a minimally informative matrix, respectively. Matrix $\mathrm{C}$ illustrates unexpected absences and presences in relation to the boundary line.

species records in sites (e.g. islands, fragments, patches, hosts) or species interactions (e.g. mutualistic or antagonistic interactions). We also use "incidence matrix" to refer both to tabular and to network representations as bipartite networks (Harary 1969).

Distributional nestedness has been documented for a variety of taxonomical groups on several spatial scales in a range of distinct biogeographical regions and habitats (Patterson 1990, Wright and Reeves 1992, Wright et al. 1998, Gaston and Blackburn 2000). Interactive nestedness seems to be a common property of mutualistic interactions, such as the interactions of plants with seed dispersers, pollinators or protective ants (Bascompte et al. 2003, Dupont et al. 2003, Ollerton et al. 2003, Guimarães et al. 2006). Nestedness also has been recorded for parasite species among conspecific host individuals (Worthen and Rohde 1996, Rohde et al. 1998). This last system represents both an interactive and a distributional matrix.

Nested structures in ecological systems can give indications of the ecological and evolutionary processes shaping interspecific interactions (Thompson 2005, Lewinsohn et al. 2006) and distribution of species among sites (Lomolino 1996). Additionally, nestedness can be a useful tool for species preservation and to establish priorities for conservation in fragmented landscapes (Patterson 1987, Boecklen 1997, MartinezMorales 2005). Indeed, recent studies suggest that nested plant-animal networks are more robust to environmental perturbation (Fortuna and Bascompte 2006).

In this paper, we address two conceptual inconsistencies related to nestedness. First, we show that the thermodynamic analogy related to matrix temperature, a metric widely used to quantify the degree of nestedness, is not valid, and we propose a new concept for matrix temperature based on its calculation. Second, we demonstrate that "anti-nestedness", a term increas- ingly used for matrix patterns that depart from nestedness, actually describes several distinct biological patterns. Thus, anti-nestedness should be avoided in favor of more meaningful concepts that describe distinct departures from nestedness. Here we will not review the measurement of nestedness and the use of appropriate metrics or null models (for these issues, see Wright and Reeves 1992, Cook and Quinn 1998, Brualdi and Sanderson 1999, Jonsson 2001, RodríguezGironéz and Santamaría 2006, Greve and Chown 2006). We will concentrate on conceptual questions and their possible consequences for data interpretation.

\section{Matrix temperature: an inadequate analogy}

The degree of nestedness of a given matrix can be assessed through different metrics (Cutler 1994, 1998, Wright et al. 1998, Brualdi and Sanderson 1999). However, several metrics are sensitive to matrix size, form and/or fill (Wright et al. 1998). This hinders comparison of nestedness among different assemblages. Wright et al. (1998) assert that these problems are less severe for matrix temperature, $\mathrm{T}$, a metric developed by Atmar and Patterson (1993) (but see RodríguezGironéz and Santamaría 2006, Greve and Chown 2006). The $T$ metric has become established as the standard measure of the degree of nestedness in studies of species distribution among sites (Fischer and Lindenmayer 2002) and of ecological interaction networks (Bascompte et al. 2003). Although we concur that $\mathrm{T}$ is a useful metric to describe nestedness, we take exception to its conceptual foundations.

Matrix temperature measures how much the incidence matrix departs from perfect nestedness. The calculation of this metric involves computational 
procedures to reorganize the incidence matrix into a state of "maximum packing", which means that the rows and columns are reordered so that nestedness is maximized, using different algorithms (Atmar and Patterson 1993, Rodríguez-Gironés and Santamaria 2006). The "packed" matrix conventionally shows rows with more presences at the top of the matrix and columns with more presences at the left side (Fig. 1). Then a boundary line is calculated to depict the expected distribution of presences if the matrix were perfectly nested (Atmar and Patterson 1993). Absences to the top and left of the line are defined as unexpected in a perfectly nested arrangement, and so are presences below and to the right (Fig. 1c). The matrix temperature is calculated as the sum of squared deviations from the boundary line of unexpected presences and absences divided by the maximum value possible for the matrix, multiplied by 100 . Thus, $T$ is a standardized (i.e. non-dimensional) index of how much the matrix departs from the perfectly nested state, combining the number and distance of unexpected presences and absences from the boundary line. In fact, $\mathrm{T}$ is a percentage measure (Rodríguez-Gironéz and Santamaría 2006).

Atmar and Patterson (1993) coined the term matrix temperature in analogy to Ludwig Boltzmann's proposition that heat is equivalent to disorder and, additionally, to Shannon's (1948) definition of entropy as an informational measure of unexpectedness. Indeed, although $\mathrm{T}$ is a standardized index, Atmar and Patterson (1993) define an unnecessary unit for $\mathrm{T}$, degrees, to reinforce the analogy. We contend that the incorporation of these terms from physics and information theory into the analysis of incidence matrices causes conceptual problems for the understanding of matrix structure. The thermodynamic analogy is applied for instance in the statement "At $100^{\circ}$, no discernible extinction order remains; the presence-absence matrix has become a free-gas" (Atmar and Patterson 1993). However, differently from what many authors state (FernándezJuricic 2002, Fischer and Lindenmayer 2002, Heino and Soininen 2005 Martínez-Morales 2005), matrix temperature does not measure presences as expected by the thermodynamical analogy of Atmar and Patterson (1993). According to their calculus of $\mathrm{T}$, matrix temperature increases with the proportion of unexpected absences and presences in the matrix with respect to perfect nestedness. Therefore, $\mathrm{T}=100$ stands for the maximum proportion of unexpected presences and absences regardless of rearranging rows and columns. It is important to notice that randomly allocated presences will produce a matrix in which $\mathrm{T}<100$ (Rodríguez-Gironéz and Santamaría 2006). Since a matrix with temperature close to 100 cannot be randomly filled, the concept of maximally disordered matrix of Atmar and Patterson (1993) becomes in- applicable. Consequently, the analogy with some features of thermodynamical or information systems, such as heat and entropy, is inappropriate and unnecessary.

We propose that a more precise definition of matrix temperature according to what is actually calculated should be "a measure of the symmetry in the distribution of unexpected absences and presences, respectively, on both sides of the perfect nestedness boundary line". When $T=0$, there is no symmetry, while $T=100$ means the highest symmetry of unexpected absences and presences at both sides of the boundary line.

\section{Anti-nestedness: a non-concept}

Anti-nestedness was first used by Wright et al. (1998) to designate "species communities that were more heterogeneous than expected by chance", but the same term designates a number of distinct structures in the literature (Table 1, Fig. 2). Since a perfectly nested matrix results in $T=0$, we can expect a value of $T$ very close or equal to 100 in a perfect anti-nested matrix. Thus, the assertion of Atmar and Patterson (1993) that at $\mathrm{T}=100$ a matrix has maximally disordered presences, can be interpreted as a non-explicit concept of anti-nestedness. We will call this the random model of anti-nestedness.

The first explicit and unambiguous concept of antinestedness appears in Poulin and Guégan (2000), for whom anti-nestedness is a departure from nestedness in which species are always absent from sites (in their case, host individuals) richer than the most depauperate one in which they occur. The second explicit usage of antinestedness corresponds to a perfect checkerboard (sensu Diamond 1975), a pattern in which the distribution of presences is exactly complementary between adjacent rows and columns. Jonsson (2001; see also Dupont et al. 2003, Lázaro et al. 2005) stated that at $T=100$, a matrix has a checkerboard pattern. Yet another concept of antinestedness, called high-turnover, was proposed by Leibold and Mikkelson (2002). According to them, an anti-nested pattern occurs when there are more replacements of presences than would be expected by chance. Note that a pure checkerboard pattern can be rearranged into a matrix with two compartments (Gotelli 2000, Lewinsohn et al. 2006), and thus it may be reduced to a particular instance either of a compartmented matrix, or of the high-turnover model. However, if the sequence of rows and columns has any spatial or biological significance, then the checkerboard arrangement will also be meaningful as a distinct pattern; for this reason, and since it is well established in the literature, we retain it as a distinct definition, to be evaluated with the others.

Although other variants are found in the literature, the concepts above illustrate the gamut of concepts that are called anti-nested. 


\section{Properties of different anti-nested concepts}

We examined some structural properties of these distinct anti-nested representations to assess more formally the difference among the definitions of anti-nestedness and thus to evaluate the applicability of this concept. To facilitate comparisons of the distinct concepts of antinestedness, we illustrate both matrix and network representations of these concepts using the same dimension of 15 rows by 5 columns (Fig. 2). We did not attempt to depict the first usage of the term anti-nested by Wright et al. (1998) because their concept is vague and does not permit an unequivocal representation.

We initially compared the other four anti-nested models by contrasting some general properties and relationships derived from these four concepts (Table 1). To evaluate further the differences among the anti-nestedness models, we assessed two additional properties: equality and overlap. We defined equality as the percentage of pairs of columns (or rows) with identical number of presences. Overlap was defined as the percentage of presences recorded in less filled columns (or rows), that are also present in more filled columns, for all paired combinations of columns.

To evaluate whether a matrix is significantly less nested than expected by chance, we used four null models and performed the analysis in the $\mathrm{C}^{++}$ program ANINHADO (Guimarães and Guimarães 2006) based on the original code of the NTC program of Atmar and Patterson (1995). The four null models used were randomized according to the following rules: (1) the probability of a presence in a given cell is constant and estimated from data as the overall proportion of occupied cells (this null model is practically identical to that of NTC); (2) the number of presences in a given row is estimated from data as the proportion of occupied cells within the row; (3) the number of presences in a given column is estimated from data as the proportion of occupied cells within the column, and (4) the probability of a given cell being occupied is the average of the probabilities of occupancy of its row and column (Bascompte et al. 2003).

Since the calculus of nestedness degree by the $T$ metric of Atmar and Patterson (1993) seems to be less affected by fill than by matrix dimensions (Wright et al. 1998, Greve and Chown 2006), we standardized all comparisons among the models using matrices with the same dimensions. Poulin and Guégan's (2000) model of non-inclusiveness with unequal sizes requires at least 55 rows for 10 columns, the dimension chosen to compare the value and significance of nestedness degree, and the mean number of presences in rows and columns.

To compare the anti-nested models as bipartite networks (Jordano et al. 2003, Lewinsohn et al. 


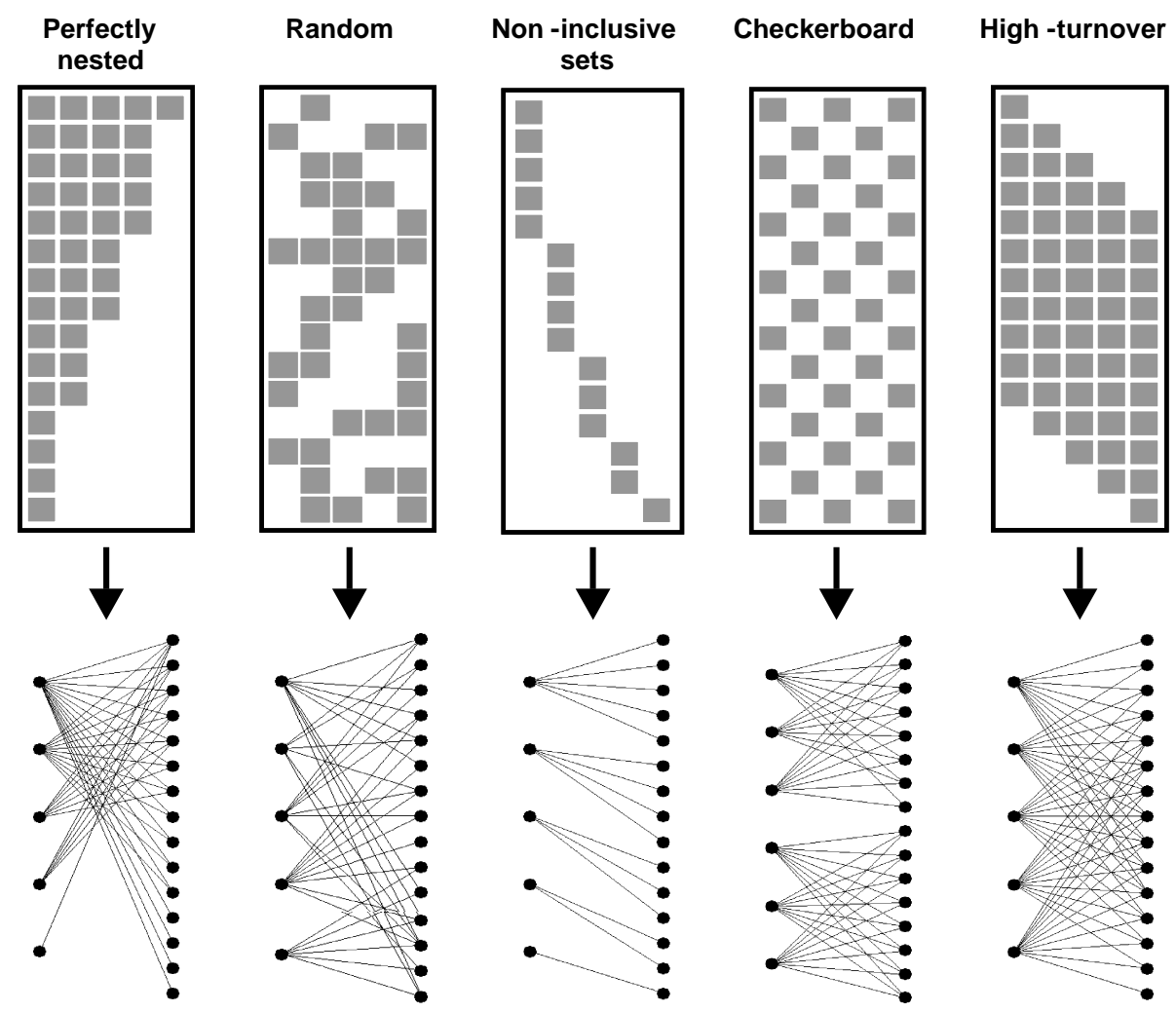

Fig. 2. Matrix and network representations of perfectly nested and four anti-nested models based on distinct concepts. All models have the same dimensions ( 5 columns and 15 rows), although fills differ.

2006), we used three properties best visualized as networks: (1) number of components, i.e. the number of non-connected sub-networks or discrete compartments; (2) the size of the largest component, i.e. the number of nodes (columns and rows) that constitute the component with the largest number of nodes, (3) average degree (and standard deviation), i.e. the average number of links (presences) per node. As we are dealing here with bipartite networks of two exclusive sets, we compare average degree separately for columns and rows. To visualize both matrix and network properties, we depicted an idealized representation for each antinested model (Fig. 2). An initial comparison of the figures of each model showed us clearly that they present distinct properties, such as the number of components and connectance.

The four anti-nested models showed marked differences in their level of nestedness (T-100) (Table 2). The difference between the highest (the non-inclusive model) and the lowest nestedness degree (the checkerboard model) was approximately 60 (Table 2 ). Only checkerboard and the sparse high-turnover models were significantly less nested than expected by any null model (Table 2). The remaining models did not show higher levels of nestedness degrees than expected by any null model, hence are not distinguishable from randomly filled matrices of similar dimensions.

These models also varied markedly in other properties such as number of components and average degree (Table 3). These properties have important consequences for the dynamics of the studied system (see Albert and Barábasi 2002 for a review in the context of complex networks). For example, the effects of environmental disturbance may not propagate through distinct components in an interactive network, since there is no connection between their species groups. Moreover, differences in degree lead to the idea that different species or sites may be more important than others to the overall stability of the system (Jordano et al. 2003, Fortuna and Bascompte 2006). Note that, since $\mathrm{T}$ is a single metric, it cannot capture all aspects of matrix properties. However, the highly different matrices that are now called anti-nested do not have any feature in common, at least from the measures examined here.

Since distinct kinds of structured incidence matrices are less nested than expected by chance, a matrix that fits this condition (Wright et al. 1998, Poulin and Guégan 2000) cannot be unambiguously assigned to a specific anti-nested concept. Therefore, we suggest that future studies on distributional and interactive species 
Table 2. Some matrix and network properties of four models of anti-nestedness based on distinct concepts. CRO $=$ completely random with constant cell occupancy probability; FRT = fixed row totals; FCT = fixed column totals; APO =average probability of occupancy. The P-values correspond to the probability of a matrix having a nestedness degree less than expected by chance according to each null model. All matrices have 55 rows by 10 columns. The nestedness degree for the maximum random model is the mean value $( \pm S D)$ for 1000 randomly generated matrices. The nestedness degree varies from 0 (no nestedness) to 100 (maximum nestedness).

\begin{tabular}{lccrrrr}
\hline Anti-nested model & Fill $(\%)$ & $\begin{array}{c}\text { Nestedness } \\
\text { degree }(100-\mathrm{T})\end{array}$ & & P-values for each null model \\
\cline { 3 - 7 } & & & CRO & FRT & FCT & APO \\
\hline Maximum randomness & 50 & $36.03 \pm 3.9$ & 0.546 & 0.760 & 0.934 & 0.148 \\
Non-inclusive sets of unequal sizes & 10 & 62.95 & 0.958 & 0.998 & 0.630 & 0.830 \\
Checkerboard presences & 50 & 3.19 & $<0.001$ & $<0.001$ & $<0.001$ & $<0.001$ \\
Highly filled high turnover* & 83.60 & 48.69 & 0.548 & 0.966 & 1.000 & 0.192 \\
Poorly filled high turnover* & 18.18 & 15.69 & $<0.001$ & $<0.001$ & $<0.001$ & $<0.001$ \\
\hline
\end{tabular}

* in order to test the effect of fill, we used two alternative models of high turnover

Table 3. Some network properties of five idealized models of anti-nestedness based on distinct concepts. All matrices have 55 rows by 10 columns. See Table 2 for further details.

\begin{tabular}{lcccc}
\hline Anti-nested model & $\begin{array}{c}\text { No. of } \\
\text { components }\end{array}$ & $\begin{array}{c}\text { Size of the largest } \\
\text { component }\end{array}$ & $\begin{array}{c}\text { Average number of } \\
\text { presences per columns }\end{array}$ & $\begin{array}{c}\text { Average number of } \\
\text { presences per rows }\end{array}$ \\
\hline Maximum randomness & 1 & 65 & 25 & 5 \\
Non-inclusive sets & 10 & 10 & 5.5 & 1 \\
Checkerboard presences & 2 & 28 & 27.5 & 5 \\
Highly filled high turnover & 1 & 65 & 46 & 8.36 \\
Poorly filled high turnover & 1 & 65 & 10 & 1.82 \\
\hline
\end{tabular}

patterns forgo the use of "anti-nestedness" in favour of more specific concepts based on testable and comparable patterns.

\section{Conclusion}

Since the metric of matrix temperature proposed by Atmar and Patterson (1993) is in wide use in studies on distributional and interactive nestedness, we propose a concept to revise the underpinnings of this metric. More specifically, we argue that (1) there is no reason to maintain the analogy between matrix temperature and thermodynamic systems, and (2) authors should not state that when $T=100$ a matrix represents a random distribution of presences, since this is only fulfilled by matrices with symmetric (i.e. non-random) distribution of unexpected absences and presences at both sides of the boundary line.

Why has the term "anti-nested" been employed to describe so strikingly dissimilar patterns? We believe that this can be ascribed to two major motivations: (1) nestedness seems to be a widespread ecological pattern, and (2) to measure and test nestedness is easier than to measure and test the majority of other structured patterns on species distribution and species interaction, due to computational ease (several free available programs). These motivations have promoted nestedness to the status of "pattern to be tested a priori", even if several others structured species distributions or interactions also are potentially detectable in a data set (Leibold and Mikkelson 2002, Lewinsohn et al. 2006). Since matrices that are less nested than expected by chance may result from different ecological processes and show distinct structural patterns, there is no sound basis for designating "anti-nestedness" as the opposite of nestedness. By this logic, one would need anticoncepts for each structured model (e.g. anti-turnover, anti-checkerboard). It makes more sense to test each distinct non-random pattern according to its assumptions and mechanistic hypotheses.

Acknowledgements - We thank Paulo I. Prado, Miguel Fortuna, Rafael D. Loyola, Sérgio F. dos Reis, Jordi Bascompte and Gregory Mikkelson. Authors were funded by FAPESP (grant no. 03/02548-1 to MAN, no. 03/03857-8 to PRG, no. 04/15482-1 to TML) and by CNPq (grant no. $306049 / 2004$ to TML).

\section{References}

Albert, R. and Barábasi, A. L. 2002. Statistical mechanics of complex networks. - Rev. Mod. Phys. 74: 47-97.

Atmar, W. and Patterson, B. D. 1993. The measure of order and disorder in the distribution of species in fragmented habitat. - Oecologia 96: 373-382.

Atmar, W. and Patterson, B. D. 1995. The nestedness temperature calculator: a Visual Basic program, including 
294 presence-absence matrices. - AICS Research, Univ. Park, NM and Field Museum, Chicago (http://aicsresearch.com/nestedness/tempcalc.html).

Bascompte, J. et al. 2003. The nested assembly of plantanimal mutualistic networks. - Proc. Natl Acad. Sci. USA 100: 9383-9387.

Boecklen, W. J. 1997. Nestedness, biogeographic theory, and the design of nature reserves. - Oecologia 112: 123-142.

Brualdi, R. A. and Sanderson, J. G. 1999. Nested species subsets, gaps, and discrepancy. - Oecologia 119: 256-264.

Cook, R. R. and Quinn, J. F. 1998. An evaluation of randomization models for nested species subsets analysis. - Oecologia 113: 584-592.

Cutler, A. H. 1994. Nested biotas and biological conservation: metrics, mechanisms, and meaning of nestedness. - Landscape Urban Planning 28: 73-82.

Cutler, A. H. 1998. Nested patterns of species distribution: processes and implications. - In: McKinney, M. L. and Drake, J. A. (eds), Biodiversity dynamics: turnover of populations, taxa, and communities. Columbia Univ. Press, pp. 212-231.

Darlington, P. J. 1957. Zoogeography: the geographical distribution of animals. - John Wiley.

Diamond, J. M. 1975. Assembly of species communities. - In: Cody, M. L. and Diamond, J. M. (eds), Ecology and evolution of communities. Belknap Press, pp. 342444.

Dupont, Y. L. et al. 2003. Structure of a plant-flower-visitor network in the high-altitude sub-alpine desert of Tenerife, Canary Islands. - Ecography 26: 301-310.

Fernández-Juricic, E. 2002. Can human disturbance promote nestedness? A case study with birds in an urban fragmented landscape. - Oecologia 131: 269-278.

Fischer, J. and Lindenmayer, D. B. 2002. Treating the nestedness temperature calculator as a "black box" can lead to false conclusions. - Oikos 99: 193-199.

Fortuna, M. A. and Bascompte, J. 2006. Habitat loss and the structure of plant-animal mutualistic networks. - Ecol. Lett. 9: $281-286$.

Gaston, K. J. and Blackburn, T. M. 2000. Pattern and process in macroecology. - Blackwell Science.

Gotelli, N. J. 2000. Null model analysis of species co-occurrence patterns. - Ecology 81: 2606-2621.

Greve, M. and Chown, S. L. 2006. Endemicity biases nestedness metrics: a demonstration, explanation and solution. - Ecography 29: 347-356.

Guimarães, P. R. and Guimarães, P. 2006. Improving the analyses of nestedness for large sets of matrices. - Environ. Model. Soft. 21: 1512-1513.

Guimarães, P. R. et al. 2006. Assimetries in specialization in ant-plant mutualistic networks. - Proc. R. Soc. Lond. B 273: $2041-2047$.

Harary, F. 1969. Graph theory. - Perseus Books Publishing.

Heino, J. and Soininen, J. 2005. Assembly rules and community models for unicellular organisms: patterns in diatoms of boreal streams. - Freshwater Biol. 50: 567-577.

Jonsson, B. G. 2001. A null model for randomization tests of nestedness in species assemblages. - Oecologia 127: $309-313$.
Jordano, P. 1987. Patterns of mutualistic interactions in pollination and seed dispersal: connectance, dependence asymmetries, and coevolution. - Am. Nat. 129: 657677.

Jordano, P. et al. 2003. Invariant properties in coevolutionary networks of plant-animal interactions. - Ecol. Lett. 6: 69-81.

Lázaro, A. et al. 2005. Bird-made fruit orchards in northern Europe: nestedness and network properties. - Oikos 110: $321-329$.

Leibold, M. A. and Mikkelson, G. M. 2002. Coherence, species turnover, and boundary clumping: elements of meta-community structure. - Oikos 97: 237-250.

Lewinsohn, T. M. et al. 2006. Structure in plant-animal interaction assemblages. - Oikos 113: 174-184.

Lomolino, M. V. 1996. Investigating causality of nestedness of insular communities: selective immigrations or extinctions? - J. Biogeogr. 23: 699-703.

Martínez-Morales, M. A. 2005. Nested species assemblages as a tool to detect sensitivity to forest fragmentation: the case of cloud forest birds. - Oikos 108: 634-642.

Ollerton, J. et al. 2003. The pollination ecology of an assemblage of grassland asclepiads in South Africa. - Ann. Bot. 92: 807-834.

Pascual, M. and Dunne, J. A. 2005. Ecological networks: linking structure to dynamics in food webs. - Oxford Univ. Press.

Patterson, B. D. 1987. The principle of nested subsets and its implications for biological conservation. - Conserv. Biol. 1: 323-334.

Patterson, B. D. 1990. On the temporal development of nested subset patterns of species composition. - Oikos 59: $330-342$.

Patterson, B. D. and Atmar, W. 1986. Nested subsets and the structure of insular mammalian faunas and archipelagos. - Biol. J. Linn. Soc. 28: 65-82.

Pimm, S. L. 1982. Food webs. - Chapman and Hall.

Poulin, R. and Guégan, J. F. 2000. Nestedness, antinestedness, and the relationship between prevalence and intensity in ectoparasite assemblages of marine fish: a spatial model of species coexistence. - Int. J. Parasitol. 30: 1147-1152.

Rodríguez-Gironéz, M. A. and Santamaría, L. 2006. A new algorithm to calculate the nestedness temperature of presence-absence matrices. - J. Biogeogr. 33: 924-935.

Rohde, K. et al. 1998. Nestedness in assemblages of metazoan ecto- and endoparasites of marine fish. - Int. J. Parasitol. 28: 543-549.

Shannon, C. E. 1948. A mathematical theory of communication. - Bell Syst. Tech. J. 27: 379-656.

Thompson, J. N. 2005. The geographic mosaic of coevolution. - Univ. of Chicago Press.

Worthen, W. B. and Rohde, K. 1996. Nested subset analyses of colonization-dominated communities: metazoan ectoparasites of marine fishes. - Oikos 75: 471-478.

Wright, D. H. and Reeves, J. 1992. On the meaning and measurement of nestedness of species assemblages. - Oecologia 92: 416-428.

Wright, D. H. et al. 1998. A comparative analysis of nested subset patterns of species composition. - Oecologia 113: $1-20$. 